INPLASY

PROTOCOL

To cite: Lu et al. Clinical prognosis of SWI hypointense vessels in ischemic stroke patients: a systematic review and meta-analysis. Inplasy protocol 202140066. doi: 10.37766/inplasy2021.4.0066

Received: 11 April 2021

Published: 11 April 2021

Corresponding author:

Ping Lu

504257712@qq.com

Author Affiliation:

Beijing Tiantan Hospital

Affiliated to Capital Medical

University

Support: No.

Review Stage at time of this submission: Data analysis.

Conflicts of interest:

None declared.

\section{Clinical prognosis of SWI hypointense vessels in ischemic stroke patients: a systematic review and meta-analysis}

Lu, P1; Cui, L².

Review question / Objective: P: Ischemic stroke patients; I: With SWI hypointense vessels; C: Without SWI hypointense vessels; 0 : Outcomes were unfavorable functional outcome (primary, modified Rankin scale scores 3-6 or 2-6), death, intermediate clinical and imaging outcomes.; S: Cohort studies/Case control studies.

Condition being studied: In the past three decades, the mortality rate of stroke in China has risen sharply, faster than that in other countries. The most common stroke subtype was ischemic stroke, accounting for $69.6 \%$ of all stroke. This disease is caused by various causes of regional blood supply disorders in brain tissue, leading to cerebral ischemia and hypoxianecrosis, and then produce the corresponding clinical manifestations of neurological deficit.Early judgment of the prognosis of stroke patients is helpful to choose the intervention measures and timing, so as to improve the clinical outcome and further reduce the burden associated with stroke.

INPLASY registration number: This protocol was registered with the International Platform of Registered Systematic Review and Meta-Analysis Protocols (INPLASY) on 11 April 2021 and was last updated on 11 April 2021 (registration number INPLASY202140066).

\section{INTRODUCTION}

Review question / Objective: P: Ischemic stroke patients; I: With SWI hypointense vessels; C: Without SWI hypointense vessels; O: Outcomes were unfavorable functional outcome (primary, modified Rankin scale scores 3-6 or 2-6), death, intermediate clinical and imaging 
outcomes.; S: Cohort studies/Case control studies.

Condition being studied: In the past three decades, the mortality rate of stroke in China has risen sharply, faster than that in other countries. The most common stroke subtype was ischemic stroke, accounting for $69.6 \%$ of all stroke. This disease is caused by various causes of regional blood supply disorders in brain tissue, leading to cerebral ischemia and hypoxianecrosis, and then produce the corresponding clinical manifestations of neurological deficit.Early judgment of the prognosis of stroke patients is helpful to choose the intervention measures and timing, so as to improve the clinical outcome and further reduce the burden associated with stroke.

\section{METHODS}

Participant or population: We enrolled patients with ischemic stroke patients (age $\geq 18$ years) who underwent baseline brain MRI after admission. Our aim was to extract data from the report on infarct growth, cerebral hemorrhage, and short / long-term clinical outcomes. We excluded: 1. Studies that did not separately report the results of participants with and without SWI hypointense vessels; 2. Reviews, editorials, letters, case or case series reports, guidelines, technical notes, and book chapters. If data can be extracted, the meeting summary is not excluded.

Intervention: With SWI hypointense vessels.

\section{Comparator: Without SWI hypointense vessels.}

Study designs to be included: Cohort studies/Case control studies.

Eligibility criteria: We included retrospective studies that recruited AIS patients ( $\geq 18$ years) who underwent baseline MRI after admission and before intravenous thrombolysis or intravascular therapy. We aimed to extract data from the report on infarct growth, cerebral hemorrhage, and short / long-term clinical outcomes as measured by the National Institutes of Health Stroke Scale (NIHSS) or Mrs score. We excluded: 1) studies that did not report outcomes separately for patients with and without swi-pvs; 2) studies whose main purpose was to evaluate the impact of swi-pvs changes on clinical prognosis before and after thrombolysis, or to explore the association between swi-pvs and other imaging features (such as collateral circulation, state of concern or perfusion dispersion mismatch); 3) reviews, editorials, letters, case or case series reports, meetings Abstracts, guides, technical notes and book chapters.

Information sources: PubMed, Embase, CBM and the Cochrane Central Register of Controlled Trials were searched from inception to the end of March 2021.

Main outcome(s): The main outcome was an adverse functional outcome, defined by a Mrs score of 2-6 or 3-6.

Quality assessment / Risk of bias analysis: The two authors also assessed the quality of each included study based on Cochrane Collaboration's RCT tool or the NewcastleOttawa Scale (NOS) for cohort studies.

Strategy of data synthesis: Mean values with SD or median values with ranges or IQRs were collated for continuous outcomes. Pooled risk ratios (RR) with $95 \% \mathrm{Cl}$ were estimated for dichotomous outcomes using the DerSimonian and Laird random-effects model. For changes in NIHSS scores and infarct growth, the extracted data were only tabulated, given their non-normal distribution and it was not common to estimate pooled median differences through meta-analysis. In every case, a two-sided $p$ value $\leq 0.05$ was deemed significant. The percentage of variability across the pooled estimates attributable to heterogeneity beyond chance was estimated using the $I^{\star 2} 2$ statistic, and by calculating the $p$ value for heterogeneity. I*2 values of $25 \%, 50 \%$ and $75 \%$ were regarded as low, moderate and high heterogeneity, respectively. All statistical analyses were performed using Stata V.16.0. 
Subgroup analysis: According to the plan, subgroup analysis was performed on the main outcome by the treatment given to the patients or the type of SWI hypointense vessels defined by location or degree.

Sensitivity analysis: The main outcome was an adverse functional outcome, defined by a Mrs score of 2-6 or 3-6.(90-day MRS).

Country(ies) involved: China.

Keywords: SWI; Stroke; Brain ischemia; Prognosis.

Contributions of each author:

Author 1 - Ping Lu.

Author 2 - Lingyun Cui. 